OPEN ACCESS

Citation: I. Monticone (2021) Raccontare con le immagini. Lo Stop-motion come strumento per l'apprendimento collaborativo. Media Education 12(2): 83-92. doi: 10.36253/me-11427

Received: June, 2021

Accepted: October, 2021

Published: December, 2021

Copyright: @ $2021 \mathrm{I}$. Monticone. This is an open access, peer-reviewed article published by Firenze University Press (http://www.fupress.com/me) and distributed under the terms of the Creative Commons Attribution License, which permits unrestricted use, distribution, and reproduction in any medium, provided the original author and source are credited.

Data Availability Statement: All relevant data are within the paper and its Supporting Information files.

Competing Interests: The Author(s) declare(s) no conflict of interest.
Best Practices

\section{Raccontare con le immagini. Lo Stop-motion come strumento per l'apprendimento collaborativo}

\author{
Telling with images. Stop-motion as a tool for collaborative \\ learning
}

\section{ILARIA MONTICONE}

Istituto Sant'Anna, scuola primaria, Torino

ilaria.monticone14@gmail.com

\begin{abstract}
The purpose of this paper is to describe the activities carried out in a class with children of the third year of primary school (Istituto Sant'Anna, Torino) school year 2019/2020 and to test the potentialities of audiovisual and technological language in the development of knowledge and skills, like the management of a collaborative activity, the correct use of technological tools and the awarness of the project's path. Thissperimental activity starts from the hypothesis that creating a stop-motion video, focused on a disciplinary subject, can give good results both from a learning point of view and the acquisition of social and relational skills thank to the type work, mainly carried out in groups.
\end{abstract}

Keywords: communication, media, multimedia languages, stop-motion.

Riassunto. Larticolo racconta l'attività svolta con gli alunni di una classe terza della scuola primaria (Istituto Sant'Anna, Torino) nell'anno scolastico 2019/2020 e ha come oggetto la sperimentazione delle potenzialità del linguaggio audiovisivo e tecnologico nello sviluppo di competenze, conoscenze e di abilità, come la gestione del lavoro collaborativo, l'uso corretto degli strumenti tecnici, la conoscenza e consapevolezza del percorso progettuale. Tale progetto sperimentale è stato avviato in collaborazione con il centro interdipartimentale di Ricerca CINEDUMEDIA dell'Università degli Studi di Torino. Tale lavoro sperimentale parte dall'ipotesi che la creazione di un video con stop-motion, incentrato su un argomento disciplinare, possa dare buoni risultati sia dal punto di vista dell'acquisizione dei contenuti, sia nell'acquisizione di competenze sociali e relazionali, stimolate dalla tipologia di lavoro, svolta principalmente a gruppi.

Parole chiave: comunicazione, linguaggi multimediali, media, stop-motion. 
LUOGO: Scuola Primaria, Istituto Paritario San'Anna (Torino)

UTENTI: Classe terza, scuola primaria composta da 22 alunni (11 maschi e 11 femmine)

DURATA: 3 mesi (da dicembre a febbraio) - 2 pomeriggi a settimana

MATERIALI E TECNOLOGIE IMPIEGATE: Tablet, LIM, computer portatile

PRODOTTO REALIZZATO: Cartone in Stop-motion sul periodo storico che va dallo scoppio del Big Bang alla comparsa degli ominidi sulla terra.

\section{IL CONTESTO}

La sperimentazione si è svolta in una classe terza primaria dell'Istituto Sant'Anna di Torino.

L'istituto è situato nel quartiere Crocetta di Torino, a sud rispetto al centro storico cittadino: risulta, storicamente, una delle zone residenziali di maggior prestigio all'interno della città. La scuola sia per la zona di appartenenza, sia per la sua natura di scuola paritaria di indirizzo cattolico ha una popolazione scolastica di ceto medio-alto e una bassa incidenza di allievi extracomunitari, rispetto alla media cittadina.

\section{FINALITÀ E OBIETTIVI DELL'ATTIVITÀ}

Il progetto si propone di sviluppare metodologie e modelli formativi atti a garantire quella congruenza tra curricolo scolastico, esperienze di socialità e consapevolezza nell'agire mediale favorendo un apprendimento sia formale, sia informale.

Alla luce di questa premessa le finalità di questo progetto possono essere così definite:

- far acquisire ai discenti una consapevolezza sempre maggiore dell'agire mediale, Questo 'agire consapevolmente' (MIUR, 2018) nell'uso dei device tecnologici o delle risorse presentate dalla rete consentirà agli studenti di apprendere affrontando le diverse situazioni educativi in modo analitico, scomponendole nei vari aspetti che le caratterizzano e pianificando per ognuno le soluzioni più idonee;

- saper accedere alle informazioni sapendole adeguatamente selezionare, organizzare, mettere in relazione, utilizzare per gestire situazioni e risolvere problemi;

- promuovere un apprendimento centrato sulla discussione, la comunicazione, il lavoro cooperativo, la contestualizzazione dei saperi nella realtà;

Il lavoro svolto con l'utilizzo della tecnica dello Stop Motion propone ai bambini di sperimentare le potenzia- lità dei linguaggi tecnologici, con particolare attenzione a quello dell'audiovisivo, facendo uso di una didattica di tipo laboratoriale volta ad apprendere le basi della scrittura con i media.

In accordo con le finalità precedentemente esposte gli obiettivi progettuali sono:

- acquisire competenze tecniche specifiche in ambito digitale;

- utilizzare una narrazione che sfrutta il potere le immagini per raccontare un sapere disciplinare;

- rafforzare la capacità logico-cognitive, di ideazione e narrazione;

- implementare le abilità creative, artistiche ed espressive: saper coordinare tecniche di produzione manuale con tecniche narrative attraverso gli strumenti digitali;

- rafforzare le competenze trasversali, in particolare la capacità di lavorare in gruppo per obiettivi e tempi definiti e di operare in modalità problem solving.

Tra le metodologie didattiche utilizzate nella sperimentazione possiamo citare: didattica laboratoriale, discussione guidata, lavoro a gruppi, problem solving e learning by doing.

\section{LE COMPETENZE PERSEGUITE DALLATTTIVITÃ}

In generale con il termine competenza viene indicata "la capacità di far fronte ad un compito, o un insieme di compiti, riuscendo a mettere in moto ed a orchestrare le proprie risorse interne, cognitive, affettive e volitive, e a utilizzare quelle esterne disponibili in modo coerente e fecondo" (Pellerey, 2004).

- La competenza digitale fa riferimento a un sapere e a un saper fare trasversale che rispecchia le necessità cui dovrebbe far fronte la formazione odierna dei discenti: l'organizzazione della memoria, la presenza simultanea di molti e diversi codici, la compresenza di procedure logiche e analogiche, la relazione immediata tra progettazione, operatività, controllo, fruizione e produzione. Inoltre la competenza è costituita dal sapere cercare, scegliere, valutare le informazioni in rete e nella responsabilità nell'uso dei mezzi, per non nuocere a sé stessi e agli altri (MIUR, 2018).

- Le competenze sociali e civiche, che racchiudono tutte le forme di comportamento che consentono alle persone di "partecipare in modo efficace e costruttivo alla vita sociale e lavorativa" (MIUR, 2012, p. 11). Le dimensioni fondamentali di cui si sostanzia questa competenza implicano abilità come il saper lavorare in gruppo, il cooperare, il presta- 
re aiuto, sostenere chi è in difficoltà, riconoscere e accettare le differenze.

Il lavoro con lo stop-motion richiede un'attenzione particolare all'aspetto relazionale: è importante la predisposizione di un clima di classe equilibrato e cooperativo, attraverso un'educazione alla solidarietà, all'empatia ed alla responsabilità personale ed interpersonale.

- Il senso di iniziativa ed imprenditorialità, ovvero la capacità di tradurre le idee in azioni. In questo rientrano "la creatività, l'innovazione e l'assunzione di rischi, come anche la capacità di pianificare e di gestire progetti per raggiungere obiettivi" (ibidem, p. 11).

- All'interno della sperimentazione gli alunni si sono confrontati con l'ideazione di un progetto in gruppo: questo ha richiesto di saper analizzare, organizzare, gestire il compito da svolgere e di avere determinazione e motivazione nell'ottenere gli obiettivi prefissati.

- La consapevolezza ed espressione culturale, che riguarda "l'importanza e l'espressione creativa di idee, esperienze ed emozioni in un'ampia varietà di mezzi di comunicazione, compresi la musica, le arti dello spettacolo la letteratura e le arti visive (MIUR, 2012, p. 11). Si tratta della competenza che contribuisce a costruire l'identità sociale e culturale attraverso la capacità di fruire dei linguaggi espressivi e di esprimersi attraverso linguaggi e canali diversi.

Nel lavoro svolto in classe i linguaggi espressivi utilizzati sono stati di diverso tipo: quello linguistico dal punto di vista narrativo (nell'ideazione e nella scrittura della storia), e discorsivo (nello spiegare, nel persuadere, nell'informare, nel confrontare); quello creativo e rappresentativo (nella creazione dei personaggi e degli scenari) e quello sonoro (nella registrazione degli audio e nella scelta delle musiche di sottofondo).

I processi chiave chiamati in causa da questa tipologia di lavoro sono: atteggiamento positivo, interazione sociale, auto-regolazione, produzione, identificazione scopo comunicativo e comprensione.

\section{TRAGUARDI DISCIPLINARI}

Per quanto riguarda la definizione degli obiettivi disciplinari (tab.1) il progetto si è focalizzato sulle materie inerenti all'argomento trattato nel video: l'evoluzione della Terra, dal Big Bang alla comparsa degli ominidi. Tale tematica ha fornito anche l'opportunità di svolgere un lavoro interdisciplinare, poiché unisce elementi scientifici e storici.
Tabella 1. Obiettivi disciplinari relativi all'argomento a tema della sperimentazione.

\begin{tabular}{|c|c|c|c|}
\hline $\begin{array}{l}\text { Ambito } \\
\text { disciplinare }\end{array}$ & CONOSCENZE & ABILITA' & DISPOSIZIONI \\
\hline $\begin{array}{l}\text { SCIENZE/ } \\
\text { STORIA }\end{array}$ & $\begin{array}{l}\text { - Comprendere l'uso } \\
\text { della linea del tempo e } \\
\text { il concetto di durata } \\
\text { - Conoscere l'origine } \\
\text { e la formazione della } \\
\text { terra } \\
\text { - Conoscere le forme } \\
\text { di vita sulla terra } \\
\text { prima dell'uomo } \\
\text { - Conoscere le } \\
\text { caratteristiche degli } \\
\text { ominidi }\end{array}$ & $\begin{array}{l}\text { - Saper ricostruire la } \\
\text { nascita del sistema } \\
\text { solare, della Terra e } \\
\text { della vita sulla Terra. } \\
\text { - Saper collocare sulla } \\
\text { linea del tempo le } \\
\text { forme di vita che hanno } \\
\text { abitato la terra prima } \\
\text { dell'uomo. } \\
\text { - Saper descrivere le } \\
\text { caratteristiche delle } \\
\text { forme di vita che hanno } \\
\text { abitato la terra prima } \\
\text { dell'uomo. }\end{array}$ & $\begin{array}{l}\text { - Capacità di } \\
\text { autocontrollo e di } \\
\text { collaborazione con i } \\
\text { compagni } \\
\text { - Capacità di } \\
\text { confronto critico e } \\
\text { positivo con l'altro } \\
\text { - Capacità di } \\
\text { partecipare in modo } \\
\text { attivo ed esplorativo. } \\
\text { - Capacità di usare i } \\
\text { materiali con cura e } \\
\text { rispetto }\end{array}$ \\
\hline
\end{tabular}

\section{LA SITUAZIONE PROBLEMA}

Si tratta di una situazione problematica che giustifica l'inizio del percorso didattico (Castoldi, 2011) e richiede la mobilitazione di un insieme di risorse di cui il soggetto dispone; dovrebbe, inoltre, presentare la necessità di superare un ostacolo o di risolvere una situazione di tipo concreto, significativo e motivante per gli alunni. La situazione-problema presentata alla classe è stata la proposta di produrre un video, su un argomento disciplinare, da mostrare alle altre classi seconde della scuola per prepararle all'inizio dell'anno scolastico successivo, scegliendo una tipologia di prodotto più vicina all'abitudine fruitiva dei bambini: un cartone animato o un cortometraggio di animazione.

E stato inoltre spiegato loro che, se il nostro lavoro avesse ottenuto un buon esito, sarebbe potuto diventare un esempio per progetti futuri, con altre classi della loro scuola o in altri istituti.

\section{LA SCANSIONE OPERATIVA}

\subsection{Ideazione del concept}

- La spiegazione del lavoro da svolgere e indagine sulle preconoscenze. Dato che i bambini non avevano un'idea chiara di cosa fosse lo stop-motion è stata sfruttata la LIM per far vedere loro dei video realizzati con questa tecnica: alcuni realizzati dalle scuole, altri da professionisti, per far loro comprendere le potenzialità di realizzazione dello strumento.

- La somministrazione test sull'argomento didattico selezionato, ovvero il periodo che va dallo scoppio del Big Bang alla nascita della terra e la comparsa 
degli ominidi sul pianeta Terra. Il questionario si componeva di una prima parte riguardante la collocazione cronologica dei passaggi evolutivi e di una seconda parte maggiormente incentrata su aspetti contenutistici come il nome delle specie viventi e le loro principali caratteristiche in relazione all'ambiente in evoluzione.

- La suddivisione del periodo di tempo riguardante gli eventi argomento del video in cinque parti e divisione dei bambini in altrettanti gruppi di lavoro che si sono spartiti diversi ruoli (a rotazione) durante le varie sessioni di lavoro: il regista (controlla il rispetto dello storyboard durante l'esecuzione delle operazioni, coordinando le altre figure); l'operatore di macchina (scatta le fotografie); lo scenografo (muove gli oggetti di scena) e il direttore della fotografia (controlla la corretta esecuzione delle fotografie).

- Il prodotto di ciascun gruppo è diventato un capitolo del video complessivo, introducendo così il concetto di serialità. La classe ha proposto fin dall'inizio di ideare un personaggio-guida da far interagire con le varie specie viventi nel corso della nostra storia: è stato condotto un brainstorming di idee sia sulla tipologia di soggetto da scegliere come guida, sia sul nome da assegnargli. È stata scelta "Vivina la cellulina", poiché i bambini hanno identificato la cellula come il primo essere vivente ad essere comparso sulla Terra, e allo stesso tempo risultava una componente costante degli esseri viventi successivi e più evoluti.

\subsection{La scrittura della sceneggiatura}

In questa fase è stato richiesto ai vari gruppi di provare a scrivere la storia che avrebbero voluto rappresentare nella loro scena. Gli studenti sono stati invitati a organizzare e ad ampliare il soggetto che era stato assegnato, pensando ad una prima articolazione a scene e cominciando anche a prevedere una prima ipotesi di dialoghi tra i personaggi.

Questo passaggio, che richiedeva un processo di astrazione, si è presentato come una difficoltà per i bambini. Per questo motivo si è preferito richiamare la loro attenzione, e ricreare un momento di condivisione collettiva, in cui si sono evidenziate le parti che la loro storia avrebbe sicuramente dovuto percorrere, come un inizio con presentazione del personaggio, uno svolgimento (con gli elementi che caratterizzano normalmente l'interazione tra personaggi e di conseguenza le battute che avrebbero potuto pronunciare) e una conclusione.

L'attenzione dell'insegnante può essere rivolta alla verifica dell'uso corretto della forma scritta della lingua.
È anche possibile richiamare l'attenzione degli studenti sui diversi registri espressivi ad esempio tra la parte narrativa e la parte dedicata ai dialoghi.

\subsection{Lo Storyboard}

Nella creazione dello storyboard viene richiesto al gruppo di "trascodificare in immagini le scene descritte nella sceneggiatura. Si tratta di un'importantissima operazione che consente agli studenti di iniziare a vedere su carta la traduzione della loro idea di partenza (e della conseguente scrittura)" (Denicolai \& Parola, 2017, pag. 33).

Si è compiuto, quindi, un passaggio dalla narrazione dei fatti alla rappresentazione dei fatti, tenendo in considerazione la successione degli avvenimenti in ordine cronologico e le varie inquadrature che comporranno la scena del nostro video (ibidem).

I bambini, in questa fase, hanno affrontato la tematica della successione degli avvenimenti nel tempo, e hanno cominciato a costruirsi un'idea della collocazione spaziale degli avvenimenti che andranno a rappresentare. Si deve riflettere sull'entrata in scena dei personaggi, sulla loro posizione mentre parlano o mentre invece sono statici, su quale evento far accadere prima e perché.

In questa fase è stato quindi necessario lavorare con i principali concetti spazio- temporali. Trattandosi di alunni alle prime esperienze con la metodologia ho chiesto loro di realizzare un'immagine che sintetizzasse l'evento fondamentale della scena, rimandando la definizione dei momenti di transizione alle "riprese". È stato specificato di indicare, anche, le battute principali dei vari quadranti.

\subsection{Costruzione della scenografia e dei personaggi}

Nel passaggio successivo gli alunni si sono occupati della creazione dei personaggi e delle scenografie: in questa fase è importante stabilire se si vuole lavorare in due dimensioni (cioè su un piano) o in tre dimensioni (su più piani).

Da subito l'idea di poter lavorare con la pasta modellabile ha acceso l'entusiasmo dei bambini, ed è, quindi, stata scelta come materiale di lavoro.

I bambini hanno, poi, scelto l'aspetto del personaggioguida, in modo che fosse simile nell'episodio di ciascun gruppo: si sono dimostrati abili e precisi nella creazione dei vari personaggi, manifestando grande impegno, fantasia e una buona attitudine al lavoro di manipolazione.

Ogni gruppo ha poi creato con i cartoncini colorati lo scenario necessario ed adeguato al contenuto del proprio episodio. 


\subsection{Le riprese}

Per le riprese il lavoro è stato svolto con la strumentazione presente a scuola ovvero dei notebook convertibili, su cui era installato il sistema operativo Windows e l'applicazione utilizzata è stata Stop motion studio.

La procedura di creazione del video richiedeva di scattare un certo numero di foto per ogni posizione dei personaggi e mantenere ferma l'inquadratura fino alla fine della sequenza.

Il lavoro si è svolto in uno o due incontri settimanali, nell'orario pomeridiano: l'attenzione è stata focalizzata sulla necessità di creare una scenografia del loro video, che fosse ogni volta simile, il più possibile, alla volta precedente, perché non risultassero particolari cambiamenti da una sequenza di foto all'altra.

Si è poi passati al momento delle foto: visto che gli alunni erano ancora abbastanza piccoli, è stato predefinito un numero di foto da scattare per ogni posizionamento dei personaggi in scena, ossia dieci scatti. Sarebbe stato molto interessante poter ragionare sulla relazione tra l'aumentare e il diminuire del numero di scatti e la fluidità dell'immagine, ma in questo caso non è sembrato adeguato al target e al tempo a disposizione.

Una volta finiti gli scatti delle scene vere e proprie è stato riproposto il tema della serialità con lo scopo di concentrarsi sull'assegnazione del titolo e della creazione della copertina per ogni episodio.

Per spiegare il fatto che avremmo avuto bisogno di una copertina per il video complessivo e di una per ogni episodio si è deciso di citare l'esempio concreto di Tom \& Jerry. La sigla iniziale del celebre cartone si conclude con una schermata che riporta il titolo della serie e le immagini dei due protagonisti, e successivamente compare la schermata con il titolo dell'episodio.

Un procedimento simile è stato utilizzato per far capire ai discenti che ogni gruppo avrebbe dovuto anche creare i titoli di coda del proprio capitolo, per mostrare i nomi dei creatori appartenenti al gruppo.

Successivamente in modalità collettiva si sono scelti la copertina ed il titolo del cartone collettivo: la maggioranza ha scelto per "Vivina nelle prime tre ere".

L'ultima fase delle riprese ha visto il gruppo coinvolto nella registrazione degli audio: i bambini hanno ideato i dialoghi tra i personaggi e hanno assegnato le loro voci ai vari personaggi. In questa fase la soluzione ideale è stata quella di effettuare le registrazioni con l'utilizzo di auricolari, perché il suono risultasse più pulito.

\subsection{Montaggio e post-produzione}

Dopo l'esportazione delle scene girate, dal device mobile, la procedura prevede l'esportazione del materiale trasferendolo sul programma di montaggio video.

Il montaggio è stato effettuato su pc con un software di editing video gratuito, Windows Movie Maker. Idealmente si dovrebbe richiedere al gruppo di realizzare il montaggio in base allo storyboard realizzato in precedenza, completando il lavoro, con la scelta e l'inserimento delle musiche e di eventuali dialoghi.

Per mancanza di tempo a disposizione in classe e per difficoltà di organizzazione con le attrezzature la fase di montaggio e post-produzione del video è stata svolta dall'insegnante, dopo aver raccolto tutti i materiali realizzati dai gruppi.

In questa fase l'insegnante può far comprendere agli studenti come è possibile costruire senso tramite le immagini, la scena viene composta da più immagini come la frase si compone di più parole (Denicolai \& Parola, 2017).

\subsection{Restituzione e analisi}

Il risultato finale del nostro lavoro è stato un video composto da cinque episodi. Nel momento della restituzione del video alla classe, ogni gruppo ha presentato il proprio lavoro, con la proiezione del filmato. In questa fase, il pubblico (cioè il gruppo classe) ha la possibilità di commentare il prodotto, sottolineando gli aspetti che hanno colpito, il grado di comprensione del messaggio ed eventuali suggerimenti per il miglioramento del video.

Tale tipo di attività si aderisce alle nuove modalità di interazione con i media delle fasce giovani di età: essi, infatti, sono sempre più abituati ad un diverso tipo di fruizione in cui il paradigma comunicativo cambia dalla produzione 'per' la massa alla produzione 'della' massa: il pubblico non è più passivo, ma assume una valenza produttiva, in un sistema meno gerarchico e strutturato (Profita, 2001).

Sono richiamati, anche, alcuni degli aspetti che per Henry Jenkins e collaboratori (2010, p.70) rimandano al concetto di cultura partecipativa cui i giovani, anche nella vita quotidiana, prendono parte. Tra questi ad esempio le 'espressioni creative' in nuove forme (digital sampling, lo skinning e il modding, i fan video, le fan fiction, le fanzine, i mash-up). I concetti chiave sono il sostegno alla creazione e la condivisione di materiali con altri, o ancora il 'problem solving di tipo collaborativo', lavoro in gruppi, formali e informali, per raggiungere obiettivi e sviluppare nuove conoscenze (es. Wikipedia o lo spoiling).

L'insegnante, in questo passaggio, può stimolare gli allievi ad esaminare il processo che ha portato alla costruzione del video, partendo da aspetti tecnico-produttivi fino ad arrivare alle ricadute didattiche circa l'argomento trattato. 


\section{VALUTAZIONE}

Con l'intento di analizzare i risultati ottenuti dalla messa in atto di questo progetto e al fine di verificare l'attivazione di alcuni processi chiave attesi, come l'atteggiamento positivo (partecipazione; inclusione), l'interazione sociale (lavoro in gruppo; interazione con gli adulti), l'auto-regolazione (rispetto dei tempi; rispetto delle regole; rispetto del materiale), la produzione (capacità d'uso della tecnica; creatività; impegno), l'identificazione dello scopo comunicativo e la comprensione (ricerca delle informazioni; analisi delle informazioni) è stata sviluppata una rubrica valutativa basata sulle indicazioni di Castoldi (2011) e Trinchero (2012).

Sulla base dei processi chiave appena esposti è stata strutturata la rubrica valutativa (tab. 2): si tratta di un prospetto che indica e descrive i risultati attesi del processo di apprendimento e ne mette in evidenza gli aspetti rilevanti, relativi tanto alle prestazioni (prodotti) quanto ai processi coinvolti e ne indica il livello/grado di raggiungimento.

Dal punto di vista della conoscenza disciplinare, in fase preliminare, è stato somministrato ai bambini un test per valutare la preparazione della classe sul tema oggetto della sperimentazione. Il questionario si componeva di una prima parte riguardante la collocazione cronologica dei passaggi evolutivi e di una seconda parte maggiormente incentrata su aspetti contenutistici come il nome delle specie viventi e le loro principali caratteristiche in relazione all'ambiente in evoluzione.

I dati relativi al test somministrato all'inizio ed alla fine del lavoro sembrano confermare la valenza della sperimentazione. Dal test iniziale sull'argomento era risultato che le principali carenze dei bambini si potessero riscontrare riguardo la successione temporale del periodo dallo scoppio del Big Bang alla creazione della Terra e la sequenza cronologica della comparsa dei primi esseri viventi sul pianeta. Avevano avuto meno difficoltà, invece, nell'esercizio di vero/falso e nel collegamento delle definizioni ai concetti corrispondenti.

Dai risultati finali (tab. 3) si può notare come, a seguito del lavoro di creazione dell'audiovisivo sull'argomento in questione, i bambini abbiano avuto meno incertezze negli esercizi di ricostruzione dell'ordine cronologico degli eventi.

Successivamente è stato somministrato un test di autovalutazione in forma anonima: il test era strutturato su sette domande volte a indagare il livello di gradimento dei bambini circa il lavoro svolto, il grado di apprezzamento riguardo il modo di spiegare e di presentare il progetto e inoltre presentava domande che miravano a far riflettere i bambini sul proprio impegno, sull'impe- gno del proprio gruppo e sulla percezione del proprio apprendimento.

Un dato interessante, vista la giovane età dei partecipanti, è stato lo spirito autocritico con cui hanno risposto alle domande: addirittura alcuni alunni hanno ammesso di non aver sempre portato delle buone idee all'interno del gruppo e di non essersi impegnati con costanza.

\section{CONCLUSIONI}

L'approccio collaborativo, promosso da questo tipo di sperimentazione, permette a ciascuno studente di partecipare al lavoro in gruppo seguendo le proprie inclinazioni naturali e di attivare, all'occorrenza, processi di peer tutoring. Infatti, l'elaborazione di un prodotto audiovisivo con la tecnica dello stop-motion può acquisire una valenza anche dal punto di vista dell'inclusività, se si pensa alle competenze sociali, relazionali e di collaborazione insite nella metodologia proposta.

Ad esempio, si può affermare che il linguaggio audiovisivo consenta di soffermarsi su uno dei traguardi competenza (obiettivi di apprendimento derivati dalle competenze chiave), ovvero le competenze spazio-temporali di base.

Nella realizzazione del lavoro la tecnica dello stopmotion i discenti si trovano a dover padroneggiare $\mathrm{i}$ principali concetti spaziali e temporali, ad esempio la corretta distinzione fra lato destro e sinistro dell'inquadratura, la percezione dell'orientamento nei concetti di 'davanti-dietro', 'alto-basso', la comprensione della successione logica e temporale degli eventi.

Le suddette abilità nell'ambito dello spazio-tempo costituiscono un prerequisito essenziale per tutto il futuro apprendimento dello studente, tuttavia i deficit visuospaziali (che rientrano nelle sindromi non verbali) caratterizzano il quadro clinico di moltissimi soggetti con DSA. Sono definiti come "disordini che determinano un'erronea stima degli aspetti spaziali fra diversi oggetti che riguardano il rapporto tra la persona e l'oggetto, le relazioni stesse fra diversi oggetti e l'orientamento degli stimoli, associata ad una corrispondente caduta nelle capacità di memoria e di pensiero spaziale» (Benton, 1985).

Un bambino che presenta carenze in questo ambito, anche se perfettamente dotato da un punto di vista verbale, presenterà difficoltà nella maggior parte delle materie scolastiche.

In particolare, la dislessia è accompagnata da disturbi della lateralizzazione e di organizzazione spazio-temporale. L'acquisizione dello schema corporeo, in relazio- 
Tabella 2. Rubrica valutativa relativa ai prodotti e ai processi coinvolti nella sperimentazione.

\begin{tabular}{|c|c|c|c|c|}
\hline \multicolumn{5}{|c|}{ Atteggiamento positivo } \\
\hline & Eccellente & Buono & Essenziale & Parziale \\
\hline Partecipazione & $\begin{array}{l}\text { L'alunno } \\
\text { partecipa in } \\
\text { modo attivo e } \\
\text { propositivo alle } \\
\text { attività. }\end{array}$ & $\begin{array}{l}\text { L'alunno } \\
\text { partecipa in } \\
\text { modo attivo } \\
\text { alle attività, e } \\
\text { se spronato } \\
\text { propone idee } \\
\text { personali. }\end{array}$ & $\begin{array}{l}\text { Se guidato } \\
\text { l'alunno } \\
\text { partecipa alle } \\
\text { attività. }\end{array}$ & $\begin{array}{l}\text { L'alunno ha } \\
\text { difficoltà a } \\
\text { partecipare alle } \\
\text { attività, anche } \\
\text { se aiutato } \\
\text { dall'insegnante. }\end{array}$ \\
\hline Inclusione & $\begin{array}{l}\text { L'alunno } \\
\text { dimostra } \\
\text { interesse ed } \\
\text { impegno } \\
\text { nell'inclusione } \\
\text { dei compagni } \\
\text { con BES, in } \\
\text { maniera } \\
\text { autonoma. }\end{array}$ & $\begin{array}{l}\text { L'alunno } \\
\text { dimostra } \\
\text { interesse } \\
\text { nell'inclusione } \\
\text { dei compagni } \\
\text { con BES. }\end{array}$ & $\begin{array}{l}\text { L'alunno, se } \\
\text { spronato, } \\
\text { dimostra } \\
\text { impegno } \\
\text { nell'inclusione } \\
\text { dei compagni } \\
\text { con BES. }\end{array}$ & $\begin{array}{l}\text { L'alunno ha } \\
\text { difficoltà ad } \\
\text { includere i } \\
\text { compagni con } \\
\text { BES anche se } \\
\text { spronato } \\
\text { dall'insegnante. }\end{array}$ \\
\hline \multicolumn{5}{|c|}{ Interazione sociale } \\
\hline & Eccellente & Buono & Essenziale & Parziale \\
\hline $\begin{array}{l}\text { Lavoro in } \\
\text { gruppo }\end{array}$ & $\begin{array}{l}\text { L'alunno } \\
\text { dimostra di } \\
\text { interagire } \\
\text { positivamente } \\
\text { con il gruppo, } \\
\text { con spirito di } \\
\text { iniziativa e } \\
\text { autonomia. }\end{array}$ & $\begin{array}{l}\text { L'alunno } \\
\text { dimostra di } \\
\text { interagire } \\
\text { positivamente } \\
\text { con il gruppo. }\end{array}$ & $\begin{array}{l}\text { L'alunno, se } \\
\text { spronato, } \\
\text { interagisce con } \\
\text { il gruppo. }\end{array}$ & $\begin{array}{l}\text { L'alunno ha } \\
\text { difficoltà ad } \\
\text { interagire con il } \\
\text { gruppo di } \\
\text { lavoro, anche } \\
\text { se spronato } \\
\text { dall'insegnante. }\end{array}$ \\
\hline $\begin{array}{l}\text { Interazione } \\
\text { con gli adulti }\end{array}$ & $\begin{array}{l}\text { L'alunno } \\
\text { dimostra di } \\
\text { interagire } \\
\text { positivamente } \\
\text { con gli adulti, } \\
\text { con spirito di } \\
\text { iniziativa e } \\
\text { autonomia. }\end{array}$ & $\begin{array}{l}\text { L'alunno } \\
\text { dimostra di } \\
\text { interagire } \\
\text { positivamente } \\
\text { con gli adulti. }\end{array}$ & $\begin{array}{l}\text { L'alunno, se } \\
\text { spronato, } \\
\text { interagisce con } \\
\text { gli adulti. }\end{array}$ & $\begin{array}{l}\text { L'alunno ha } \\
\text { difficoltà ad } \\
\text { interagire con } \\
\text { gli adulti, } \\
\text { anche se } \\
\text { spronato } \\
\text { dall'insegnante. }\end{array}$ \\
\hline \multicolumn{5}{|c|}{ Auto-regolazione } \\
\hline & Eccellente & Buono & Essenziale & Parziale \\
\hline $\begin{array}{l}\text { Rispetto dei } \\
\text { tempi }\end{array}$ & $\begin{array}{l}\text { L'alunno, } \\
\text { autonomamente, } \\
\text { rispetta sempre i } \\
\text { tempi assegnati } \\
\text { per le varie } \\
\text { attività. }\end{array}$ & $\begin{array}{l}\text { L'alunno } \\
\text { rispetta i } \\
\text { tempi } \\
\text { assegnati per } \\
\text { le varie } \\
\text { attività. }\end{array}$ & $\begin{array}{l}\text { L'alunno ha } \\
\text { bisogno } \\
\text { dell'aiuto o } \\
\text { l'esortazione } \\
\text { dell'insegnante } \\
\text { per rispettare i }\end{array}$ & $\begin{array}{l}\text { Anche con } \\
\text { l'aiuto } \\
\text { dell'insegnante } \\
\text { l'alunno fatica } \\
\text { a rispettare i } \\
\text { tempi assegnati }\end{array}$ \\
\hline
\end{tabular}




\begin{tabular}{|c|c|c|c|c|}
\hline & & & $\begin{array}{l}\text { tempi assegnati } \\
\text { per le varie } \\
\text { attività. }\end{array}$ & $\begin{array}{l}\text { per le varie } \\
\text { attività. }\end{array}$ \\
\hline $\begin{array}{l}\text { Rispetto delle } \\
\text { regole }\end{array}$ & $\begin{array}{l}\text { L'alunno, } \\
\text { autonomamente, } \\
\text { rispetta sempre } \\
\text { le regole date } \\
\text { nelle varie } \\
\text { attività. }\end{array}$ & $\begin{array}{l}\text { L'alunno } \\
\text { rispetta i le } \\
\text { regole date } \\
\text { nelle varie } \\
\text { attività. }\end{array}$ & $\begin{array}{l}\text { L'alunno ha } \\
\text { bisogno } \\
\text { dell'aiuto o } \\
\text { l'esortazione } \\
\text { dell'insegnante } \\
\text { per rispettare le } \\
\text { regole delle } \\
\text { varie attività. }\end{array}$ & $\begin{array}{l}\text { Anche con } \\
\text { l'aiuto } \\
\text { dell'insegnante } \\
\text { l'alunno fatica } \\
\text { a rispettare le } \\
\text { regole date } \\
\text { nelle varie } \\
\text { attività. }\end{array}$ \\
\hline $\begin{array}{l}\text { Rispetto del } \\
\text { materiale }\end{array}$ & $\begin{array}{l}\text { L'alunno, } \\
\text { autonomamente, } \\
\text { rispetta sempre } \\
\text { il materiale } \\
\text { impiegato nelle } \\
\text { varie attività. }\end{array}$ & $\begin{array}{l}\text { L'alunno } \\
\text { rispetta il } \\
\text { materiale } \\
\text { impiegato } \\
\text { nelle varie } \\
\text { attività. }\end{array}$ & $\begin{array}{l}\text { L'alunno ha } \\
\text { bisogno del } \\
\text { controllo } \\
\text { dell'insegnante } \\
\text { per il corretto } \\
\text { utilizzo del } \\
\text { materiale dato } \\
\text { per le varie } \\
\text { attività. }\end{array}$ & $\begin{array}{l}\text { Anche con il } \\
\text { controllo } \\
\text { dell'insegnante } \\
\text { l'alunno fatica } \\
\text { ad utilizzare il } \\
\text { materiale dato } \\
\text { per le varie } \\
\text { attività. }\end{array}$ \\
\hline \multicolumn{5}{|l|}{ Produzione } \\
\hline & Eccellente & Buono & Essenziale & Parziale \\
\hline Capacità d'uso & $\begin{array}{l}\text { L'alunno sa } \\
\text { utilizzare stop- } \\
\text { motion, in } \\
\text { autonomia e con } \\
\text { spirito di } \\
\text { iniziativa }\end{array}$ & $\begin{array}{l}\text { L'alunno } \\
\text { riesce } \\
\text { utilizzare le } \\
\text { stop-motion. }\end{array}$ & $\begin{array}{l}\text { L'alunno riesce } \\
\text { ad utilizzare } \\
\text { stop-motion } \\
\text { solo se guidato } \\
\text { dall'insegnante. }\end{array}$ & $\begin{array}{l}\text { Anche se } \\
\text { guidato } \\
\text { dall'insegnante } \\
\text { l'alunno ha } \\
\text { difficoltà ad } \\
\text { utilizzare stop- } \\
\text { motion. }\end{array}$ \\
\hline Creatività & $\begin{array}{l}\text { L'alunno ha } \\
\text { idee creative, } \\
\text { personali e } \\
\text { innovative in } \\
\text { merito alle } \\
\text { attività. }\end{array}$ & $\begin{array}{l}\text { L'alunno ha, } \\
\text { talvolta, idee } \\
\text { creative e } \\
\text { personali in } \\
\text { merito alle } \\
\text { attività. }\end{array}$ & $\begin{array}{l}\text { L'alunno, se } \\
\text { spronato } \\
\text { dall'insegnante } \\
\text { espone le } \\
\text { proprie idee } \\
\text { personali in } \\
\text { merito alle } \\
\text { attività. }\end{array}$ & $\begin{array}{l}\text { Anche se } \\
\text { guidato } \\
\text { dall'insegnante } \\
\text { l'alunno riesce } \\
\text { con fatica ad } \\
\text { esprimere idee } \\
\text { in merito alle } \\
\text { attività. }\end{array}$ \\
\hline Impegno & $\begin{array}{l}\text { L'alunno } \\
\text { dimostra } \\
\text { costante } \\
\text { impegno ed } \\
\text { entusiasmo } \\
\text { nelle attività. }\end{array}$ & $\begin{array}{l}\text { L'alunno } \\
\text { dimostra } \\
\text { impegno e } \\
\text { talvolta } \\
\text { entusiasmo } \\
\text { nelle attività. }\end{array}$ & $\begin{array}{l}\text { L'alunno, se } \\
\text { spronato, } \\
\text { dimostra } \\
\text { impegno nelle } \\
\text { attività. }\end{array}$ & $\begin{array}{l}\text { Anche se } \\
\text { spronato, } \\
\text { l'alunno ha } \\
\text { difficoltà a } \\
\text { dimostrare } \\
\text { impegno nelle } \\
\text { attività. }\end{array}$ \\
\hline
\end{tabular}




\begin{tabular}{|c|c|c|c|c|}
\hline \multicolumn{4}{|l|}{ Comprensione } & \multirow[b]{2}{*}{ Parziale } \\
\hline & Eccellente & Buono & Essenziale & \\
\hline $\begin{array}{l}\text { Identificazione } \\
\text { dello scopo } \\
\text { comunicativo }\end{array}$ & $\begin{array}{l}\text { L'alunno } \\
\text { comprende } \\
\text { autonomamente } \\
\text { lo scopo } \\
\text { comunicativo } \\
\text { delle attività. }\end{array}$ & $\begin{array}{l}\text { L'alunno } \\
\text { comprende lo } \\
\text { scopo } \\
\text { comunicativo } \\
\text { delle attività. }\end{array}$ & $\begin{array}{l}\text { L'alunno, se } \\
\text { guidato, } \\
\text { comprende lo } \\
\text { scopo } \\
\text { comunicativo } \\
\text { delle attività. }\end{array}$ & $\begin{array}{l}\text { Anche se } \\
\text { guidato, } \\
\text { l'alunno fatica } \\
\text { a comprendere } \\
\text { lo scopo } \\
\text { comunicativo } \\
\text { delle attività. }\end{array}$ \\
\hline $\begin{array}{l}\text { Ricerca delle } \\
\text { informazioni }\end{array}$ & $\begin{array}{l}\text { L'alunno ricerca } \\
\text { informazioni } \\
\text { pertinenti alle } \\
\text { attività in modo } \\
\text { autonomo, } \\
\text { consapevole e } \\
\text { spontaneo. }\end{array}$ & $\begin{array}{l}\text { L'alunno sa } \\
\text { ricercare } \\
\text { informazioni } \\
\text { pertinenti alle } \\
\text { attività. }\end{array}$ & $\begin{array}{l}\text { L'alunno se } \\
\text { guidato e } \\
\text { spronato } \\
\text { ricerca } \\
\text { informazioni } \\
\text { relative alle } \\
\text { attività. }\end{array}$ & $\begin{array}{l}\text { Anche se } \\
\text { guidato e } \\
\text { spronato } \\
\text { l'alunno fatica } \\
\text { a ricercare } \\
\text { informazioni } \\
\text { relative alle } \\
\text { attività. }\end{array}$ \\
\hline $\begin{array}{l}\text { Analisi delle } \\
\text { informazioni }\end{array}$ & $\begin{array}{l}\text { L'alunno } \\
\text { analizza le } \\
\text { informazioni } \\
\text { pertinenti alle } \\
\text { attività in modo } \\
\text { autonomo, } \\
\text { consapevole e } \\
\text { spontaneo. }\end{array}$ & $\begin{array}{l}\text { L'alunno sa } \\
\text { analizzare le } \\
\text { informazioni } \\
\text { pertinenti alle } \\
\text { attività. }\end{array}$ & $\begin{array}{l}\text { L'alunno se } \\
\text { guidato e } \\
\text { spronato } \\
\text { analizza le } \\
\text { informazioni } \\
\text { relative alle } \\
\text { attività. }\end{array}$ & $\begin{array}{l}\text { Anche se } \\
\text { guidato e } \\
\text { spronato } \\
\text { l'alunno fatica } \\
\text { ad analizzare } \\
\text { informazioni } \\
\text { relative alle } \\
\text { attività. }\end{array}$ \\
\hline \multicolumn{4}{|c|}{ Argomento di scienze } & \\
\hline Conoscenza & $\begin{array}{l}\text { L'alunno } \\
\text { conosce e } \\
\text { organizza i } \\
\text { contenuti } \\
\text { autonomamente, } \\
\text { in modo } \\
\text { corretto e } \\
\text { completo. }\end{array}$ & $\begin{array}{l}\text { L'alunno } \\
\text { conosce e } \\
\text { organizza i } \\
\text { contenuti in } \\
\text { modo corretto. }\end{array}$ & $\begin{array}{l}\text { L'alunno, se } \\
\text { guidato, } \\
\text { organizza i } \\
\text { contenuti in } \\
\text { modo } \\
\text { abbastanza } \\
\text { corretto. }\end{array}$ & $\begin{array}{l}\text { Anche se } \\
\text { guidato, } \\
\text { l'alunno fatica } \\
\text { ad organizzare } \\
\text { le } \\
\text { informazioni. }\end{array}$ \\
\hline Esposizione & $\begin{array}{l}\text { L'alunno } \\
\text { espone i } \\
\text { contenuti con } \\
\text { precisione e con } \\
\text { il lessico } \\
\text { specifico della } \\
\text { disciplina. }\end{array}$ & $\begin{array}{l}\text { L'alunno } \\
\text { espone i } \\
\text { contenuti con } \\
\text { proprietà } \\
\text { lessicale. }\end{array}$ & $\begin{array}{l}\text { L'alunno } \\
\text { espone con } \\
\text { proprietà di } \\
\text { linguaggio } \\
\text { sufficiente. }\end{array}$ & $\begin{array}{l}\text { L'alunno } \\
\text { espone gli } \\
\text { argomenti in } \\
\text { modo confuso } \\
\text { e lacunoso. }\end{array}$ \\
\hline
\end{tabular}


Tabella 3- Report risultati test disciplinare iniziale e finale.

\begin{tabular}{|l|l|l|}
\hline \multicolumn{2}{|l|}{ Risposte corrette agli esercizi 1 e 4 } \\
\hline & Esercizio 1 & Esercizio 4 \\
\hline Gennaio & $8 / 21$ & $5 / 21$ \\
\hline Aprile & $14 / 22$ & $13 / 22$ \\
\end{tabular}

ne allo spazio, presenta lacune nell'acquisizione dei concetti spaziali, con impedimenti a mantenere regolare e costante la dinamica di movimento dello sguardo durante la lettura (il soggetto con dislessia cambia frequentemente la direzione dello sguardo senza seguire la successione degli elementi imposta dal testo, ma saltando arbitrariamente righe e parole).

Una metodologia, come quella proposta nella sperimentazione, potrebbe aiutare gli alunni con questo tipo di difficoltà: il lavoro in gruppo con i compagni permetterebbe di introdurre le proprie capacità in attività in cui si sentano a proprio agio, senza esperire situazioni di fallimento (ad esempio il disegno, la manipolazione, l'utilizzo di un device digitale, lo speakeraggio). Nel fare questo gli studenti non dovrebbero, comunque, rinunciare all'apprendimento del contenuto disciplinare, potendo contare sull'aiuto e la collaborazione dei compagni in quei campi dove presentano delle difficoltà (come la lettura di testi, o la ricerca di informazioni).

$\mathrm{Si}$ è potuto osservare, da quanto esposto, che in questa sperimentazione viene richiamato un approccio di peer-education anzi, nel caso specifico, di peer-production (Benkler, 2003; Benkler, Shaw \& Mako Hill, 2015). Questo tipo di pratica consente, inoltre, di allenare gli studenti a un utilizzo critico del mezzo tecnologico e a un atteggiamento attivo nei confronti dell'apprendimento che si svolge in un ambiente predisposto alla collaborazione con $\mathrm{i}$ pari, seguendo una logica di learning-by-doing.

\section{BIBLIOGRAFIA}

Benton, A.L., Costa, L., \& Spreen, O. (1985). Studies in neuropsychology: selected papers of Arthur. Oxford University.

Benkler, Y. (2003). Freedom in the Commons: Towards a Political Economy of Information, 52 Duke Law Journal 1245-1276 (2003). https://scholarship.law.duke. $\mathrm{edu} / \mathrm{dlj} / \mathrm{vol} 52 / \mathrm{iss} 6 / 3$

Benkler, Y., Shaw, A., \& Mako Hill, B. (2015). Peer production: a modality of collective intelligence. In T. Malone \& M. Berstein (eds.), Handbook of Collective Intelligence (pp. 175-204), MA: MIT Press.
Castoldi, M. (2011). Progettare per competenze. Percorsi e strumenti. Carocci.

Denicolai, L., \& Parola, A. (2017). Scritture mediali. Riflessioni, rappresentazioni e proposte media educative. Mimesis.

Jenkins, H., Purushotma, R., Weigel, M., Clinton, K. \& Robison, A. J. (2010). Culture partecipative e competenze digitali. Media education per il XXI secolo. Edizioni Angelo Guerini.

MIUR. Ministero dell'Istruzione, dell'Università e Ricerca (2012), Indicazioni nazionali per il curricolo della scuola dell'infanzia e del primo ciclo d'istruzione. http://www.indicazioninazionali.it/wp-content/ uploads/2018/08/decreto-ministeriale-254-del-16-novembre-2012-indicazioni-nazionali-curricolo-scuolainfanzia-e-primo-ciclo.pdf

MIUR. Ministero dell'Istruzione, dell'Università e Ricerca. (2018). Indicazioni Nazionali e Nuovi Scenari. http://www.indicazioninazionali.it/wp-content/ uploads/2018/08/Indicazioni-nazionali-e-nuovi-scenari.pdf

Pellerey, M. (2004). Le competenze individuali e il Portfolio. La Nuova Italia, Firenze.

Profita, G. (2001). L'industria audiovisiva italiana ed europea alle soglie della rivoluzione digitale. Franco Angeli.

Trinchero, R. (2012). Costruire, valutare, certificare competenze. Proposte di attività per la scuola. Franco Angeli. 